\title{
SELECTION METRICS FOR COOPERATIVE MULTIHOP RELAYING *
}

\author{
Jonghyun Kim and Stephan Bohacek \\ Department of Electrical and Computer Engineering \\ University of Delaware \\ Newark, DE 19716 \\ kim,bohacek@eecis.udel.edu
}

Keywords: Cooperative relaying, MANETs, routing

\begin{abstract}
Cooperative relaying enables nodes to actively cooperate to deliver packets to their destination. The bestselect protocol (BSP) implements a type of cooperative relaying that generalizes single path routing with sets of nodes (relay-sets) replacing the concept of a single node relay. Thus, while in traditional single path routing, packets hop from node to node, in BSP, packets hop from relay-set to relay-set. Through the exchange of channel gain information between relay-sets, the best node within a relay-set is selected to transmit the data packet on behalf of the entire relay-set. The node selected depends on the metric used. Any metric that can be posed in a dynamic programming framework can be used. In this paper, performance gains from a number of selection metrics are investigated. Specific selection metrics include maximizing the minimum channel gain along the path, minimizing end-to-end delay, minimizing the total power, and minimizing the total energy. It will be shown that BSP can achieve significant gains in all of these metrics.
\end{abstract}

\section{Introduction}

In traditional multihop wireless data networks, route search and packet forwarding are separated; first a route is found, and then packets are forwarded along the route. In the case that multipath routing is employed, the situation is similar, but a set of paths are found, and then, packets

* This work was prepared through collaborative participation in the Collaborative Technology Alliance for Communications and Networks sponsored by the U.S. Army Research Laboratory under Cooperative Agreement DAAD19-01-2-0011. The U.S. Government is authorized to reproduce and distribute reprints for Government purposes notwithstanding any copyright notation thereon. 
are forwarded along each route either probabilistically, or the routes are used as precomputed backups [Marina and Das, 2003]. In any case, in traditional routing, nodes act alone to forward the packet to its next hop. In cooperative relaying, a group of nodes act together to forward a packet. While several variants of cooperative relaying are possible, one approach is to generalize the single node that forwards the packet to a set of nodes that cooperate (see [Zhao and Valenti, 2005] for an alternative approach). Such a set of nodes is called a relay-set. Thus, while traditional networking forwards packets from node to node, this form of cooperative relaying forwards packets from relay-set to relay-set. Within the relay-set paradigm, there are also many possible approaches. For example, in some cases, a number of nodes transmit the same or different parts of the packet. In such cases, the total transmission power used to transmit the data packet between two relay-sets is distributed among a number of node [Sendonaris et al., 2003a]. However, in [Luo et al., 2004], it was shown that in the case of two-hop paths, if the channels are known, then the optimal approach is to allocate all power to the best node pair. Such an approach is known as best-select relaying.

Best-select protocol (BSP) is a multihop extension of best-select relaying. Hence, BSP makes extensive use of channel measurements and attempts to select the best path. A distinguishing feature of BSP is that it is highly dynamic and finds paths on a per packet basis. The path that a packet follows depends on instantaneous and smoothed channel gain measurements. As a result of the highly reactive nature of BSP, one might expect that BSP would be able to find paths that provide substantially better performance over a static path. This paper examines if this hypothesis is true.

The performance improvement attained depends on the metric used to select paths. This paper considers the performance improvements that result from maximizing the minimum channel gain along the path, minimizing end-to-end delay, minimizing the total power, and minimizing the total energy. It will be shown that BSP can achieve significant gains in all of these metrics.

This investigation examines these performance gains in two different scenarios. Specifically, we consider an idealized version of BSP when the nodes are in an urban area, and a QualNet [Scalable Network Technologies, 2005] implementation of BSP when nodes are in an urban area. The urban area simulations utilize channel gains from performing ray-tracing on a map of an urban area [Sridhara et al., 2005]. Thus, these channel gains are similar to those that would be found in an urban deployment. In each scenario, two different node densities are examined, sparse and dense. 


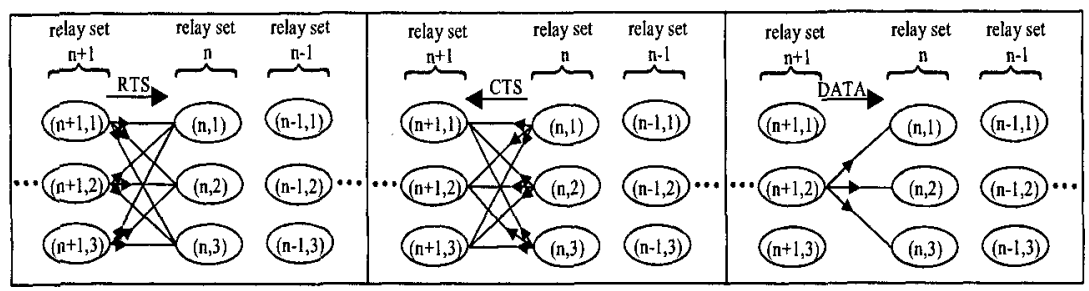

Figure 1. Best-Select Protocol

The paper proceeds as follows. In the next section a brief overview of BSP is provided. In Section 3, the methodology for evaluating the performance gains is discussed. In Section 4 the different selection metrics are evaluated. Finally, Section 5 provides a summary of the results and concluding remarks.

\section{An Overview of Best-Select Protocol (BSP)}

BSP groups nodes into relay-sets. The relay-set that is $n$ hops from the destination is referred to as the $n$-th relay-set. The $i$-th node within the $n$-th relay-set is denoted by $(n, i)$. The nodes within the $n$-th relayset cooperate with the nodes within the $(n-1)$-th relay-set to determine which node in the $n$-th relay-set should transmit the data packet. Specifically, the nodes within the $n$-th relay-set transmit a RTS packet to the nodes in the $(n-1)$-th relay-set. These transmissions occur simultaneously using CDMA with each node using a different code. Each node in the $(n-1)$-th relay-set receives all the RTSs and records the channel gains over each channel. We denote the channel gain from node $(n, i)$ to node $(n-1, j)$ as $R_{(n, i),(n-1, j)}$. Assuming that the channel is idle, all the nodes in the $(n-1)$-th relay-set transmit a CTS simultaneously using CDMA. These CTS packets contain the just measured channel gains along with other channel gain information. Each node in the $n$ th relay-set receives these CTSs along with the embedded channel gain information. Since all nodes have received the same information, they are able to make the same decision as to which node is best suited to transmit. This node then transmits the data packet using the entire bandwidth. Figure 1 illustrates the approach.

The decision as to which node is best suited to transmit does not only depend the channel gains $R_{(n, i),(n-1, j)}$, but also on the downstream channel gains, $R_{(n-1, j),(n-2, k)}, R_{(n-2, k),(n-3, l)}$, etc. This amount of channel gain information cannot be economically included into the CTS packets. Instead, the downstream channel information is encapsulated into a scalar, which we denote as $J$. Specifically, the relevant downstream 
channel information from node $(n, i)$ is denoted $J_{(n, i)}$ and depends on the selection metric. In this paper we explore several different objectives, and hence $J$ will take many different meanings. However, in all cases, $J$ will encapsulate the downstream channel information.

\section{Methodology}

In order to model multihop mobile network in a more realistic urban area, a realistic propagation and mobility tool is used. This tool is described in [Sridhara et al., 2005]. Here, the Paddington area of London is considered.

Two different approaches and two different scenarios are used to evaluate the selection metrics. We refer to the approaches as urban idealized BSP and urban implemented BSP.

Idealized simulation of BSP is performed with Matlab. Once the channel gains from propagation tool are known, the selection metrics can be investigated. A source and destination are selected at random, and a least-hop path between these nodes is found. If there exists multiple least-hop paths, one is selected, and if there are no paths, a new source and destination are selected. The nodes within the least-hop path make up the initial relay-sets. If a node is able to communicate with a node in the $(n+1)$-th relay-set and a node in the $(n-1)$-th relay-set, then the node joins the $n$-th relay-set. The best path (in terms of the metric under consideration) among all paths that are made up of nodes within the relay-sets is found. The value of the metric for the best path and the value for the initial, randomly selected path are recorded. Next, time is increased by one second. As a result, the nodes may move and new channel gains result. If the nodes which had composed the least-hop path are still connected, then the process is repeated. The metrics are repeatedly evaluated until the least-hop path breaks or the simulation ends (300 seconds). Since this approach is able to always correctly build the relay-sets and always uses the correct value of the selection metric, we call this an idealized BSP.

Packet simulation of BSP is performed with QualNet [Scalable Network Technologies, 2005]. To evaluate the selection metrics, the same source-destination pairs, mobility, and channel gains used by the urban idealized BSP are used. The implementation of BSP is much like the idealized BSP in the sense that first a least-hop path is found. This path is then enhanced. Specifically, every time a an RTS-CTS exchanges occurs as described in the previous section, if a node can hear some node in the $(n+1)$-th relay-set and a node in the $(n-1)$-th relay-set, then the node joins the $n$-th relay-set. The value of the metric is determines in the same way as it is in the idealized BSP case. The main difference 
between the idealized BSP and the implement BSP is that the relay-sets grow more slowly.

\section{Selection Metrics}

Like routing metrics, cooperative relaying allows the selection of links according to different metrics. However, the highly dynamic nature of cooperative relaying allows new metrics to be explored. To see this, consider the channel gain. While it is possible to use the channel gain for selecting a path that will be used statically (or until it breaks), it has been shown that channel gain is only a marginal predictor of the quality of the path [Bohacek et al., 2005]. The reason for this is that the channel gain may rapidly vary and is difficult to predict, especially on the time-scales relevant for routing.

On the other hand, BSP is able to quickly adjust the way in which packets are delivered. Specifically, the exact path a packet takes is determined only as the packet is being sent through the network. Thus, BSP is able to react quickly to changes in the channel gains. The question addressed by this paper is which metrics can be used and what is the impact of using best-select with these metrics.

\subsection{Maximizing the Minimum Channel Gain Along the Path}

The received signal strength is the product of the transmission power and the channel gain. Thus for a fixed transmission power and noise, the SNR is a linear function of the channel gain. Thus, a high channel gain allows for a low transmission error, lower transmission power, and/or higher data rate. Here, the selection metric finds the path that has the largest minimum channel gain. That is, for each hop along the path, the channel gain is evaluated. The quality of the path is taken to be the smallest channel gain along the path. The link with the smallest channel gain can be thought of as the bottleneck of the path. Hence, we seek to select the path with the best bottleneck.

Define $J_{(n, i)}$ to be the minimum channel gain over the best path from node $(n, i)$ to the destination. Then the following holds

$$
J_{(n, i)}=\max _{j}\left(\min \left(R_{(n, i),(n-1, j)}, J_{(n-1, j)}\right)\right)
$$

where the maximization is over all nodes in the $(n-1)$-th relay-set.

In order to evaluate this metric in the idealized case, we can simply examine $J_{(N, 1)}$, where there are $N$ hops between the source and destination. However, in the implementation of BSP, we evaluate the selection metric for each packet delivered by taking the minimum value of $\max _{j}\left(\min \left(R_{\left(n, i_{n}^{*}\right),(n-1, j)}, J_{(n-1, j)}\right)\right)$, where node $\left(n, i_{n}^{*}\right)$ is selected to 
transmit the node. More specifically, suppose that the nodes that are selected to transmit the packet are $(N, 1),\left(N-1, i_{N-1}^{*}\right),\left(N-2, i_{N-2}^{*}\right), \ldots$ Then the value of the metric is

$$
\min _{n} \max _{j}\left(\min \left(R_{\left(n, i_{n}^{*}\right),(n-1, j)}, J_{(n-1, j)}\right)\right) .
$$

Note that in this case, the value of the metric depends of which nodes are able to decode the packet and hence the minimum gain experienced may be different from $J_{(N, 1)}$. To see this how this can occur, suppose that node $(n, i)$ is selected to transmit the data packet and $j^{o}=\arg \max _{j}\left(\min \left(R_{\left(n, i_{n}^{*}\right),(n-1, j)}, J_{(n-1, j)}\right)\right)$. This means that the best next hop is $\left(n-1, j^{\circ}\right)$. However, there may be some other node $j^{+}$ such that $J_{\left(n-1, j^{+}\right)}>J_{\left(n-1, j^{\circ}\right)}$, but $\min \left(R_{\left(n, i_{n}^{*}\right),\left(n-1, j^{\circ}\right)}, J_{\left(n-1, j^{\circ}\right)}\right)>$ $\min \left(R_{\left(n, i_{n}^{*}\right),\left(n-1, j^{+}\right)}, J_{\left(n-1, j^{+}\right)}\right)$. Thus, node $\left(n-1, j^{+}\right)$is not the best next hop. However, if node $\left(n-1, j^{+}\right)$is able to decode the packet, then, since $J_{\left(n-1, j^{+}\right)}>J_{\left(n-1, j^{\circ}\right)}$, node $\left(n-1, j^{+}\right)$is better suited to transmit than node $\left(n-1, j^{*}\right)$. On the other hand, since $R_{\left(n, i_{n}^{*}\right),\left(n-1, j^{\circ}\right)}>$ $R_{\left(n, i_{n}^{*}\right),\left(n-1, j^{+}\right)}$, node $\left(n-1, j^{+}\right)$is less likely to decode the packet than node $\left(n-1, j^{o}\right)$ (this why node $\left(n-1, j^{o}\right)$ is the best next hop). But in the cases that node $\left(n-1, j^{+}\right)$is able to correctly decode the packet, then its ability to deliver the packet to the designation should be utilized, which is what BSP does. The ability to use relays that, while not the most reliable, can sometimes act is good relays is called opportunistic relaying and is a distinctive feature of BSP that is not shared with traditional routing protocols. However, the metric discussed here does not reflect the possibility of opportunistic relaying, and hence the value of the metric for the implemented BSP might be larger than for the idealized case. Figure 2 shows that this difference is especially noticeable for small relay-set size. Below, some metrics consider the possibly of opportunistic relaying and some do not.

The performance of this selection metric is shown in the left-hand plot in Figure 2. In general, BSP is able to provide significantly higher minimum channel gain than the least-hop routing. For example, two orders of magnitude improvement is not uncommon for the idealized and implemented urban cases.

\subsection{Minimizing the End-to-End Delay}

Here we focus on the expected value of the end-to-end delay, i.e., the sum of each transmission delay (we do not consider queuing or processing delay).

It is assumed that if a packet is lost due to transmission error, the end-to-end delay is $T$, where $T$ is a large number. The motivation for 

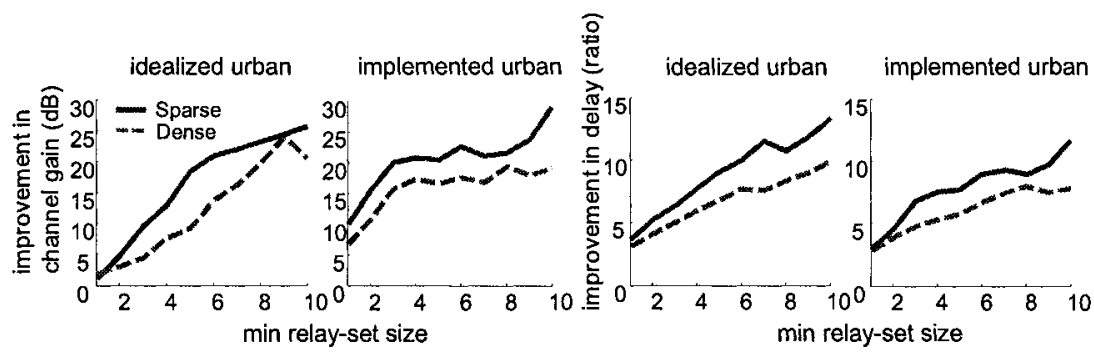

Figure 2. Left : Min Channel Gain. The average ratio of the minimum channel gain along the path with BSP to the minimum channel gain along the path with least-hop routing. Right : Delay. The average ratio of the end-to-end delay of least-hop routing to the end-to-end delay with BSP.

this is that if a packet is lost, then the transport layer will be forced to retransmit, resulting in a large delay. $T$ is further discussed later.

Here $J_{(n, i)}$ is defined as the expected sum of the transmission delays from node $(n, i)$ to the destination. Furthermore, let $J_{(n, i)}(B)$ be the expected delay from node $(n, i)$ to the destination if node $(n, i)$ transmits at bit-rate $B$. Let $f(V)$ be the probability of transmission error when the channel gain is $V$, and let $J_{(n, i)}$ be the probability of successfully delivering the packet to the destination from node $(n, i)$. Furthermore, let $\mathcal{I}$ be an ordering of the nodes in the $(n-1)$-th relay-set such that $J_{(n-1, \mathcal{I}(1))} \geq J_{(n-1, \mathcal{I}(1))} \geq \cdots$. Then,

$$
\begin{aligned}
& J_{(n, i)}(B)=\frac{\text { packet size }}{B}\left\{f\left(R_{(n, i),(n-1, \mathcal{I}(1))} X, B\right)\right. \\
& \left.+\left(1-f\left(R_{(n, i),(n-1, \mathcal{I}(1))} X, B\right)\right) f\left(R_{(n, i),(n-1, \mathcal{I}(2))} X, B\right)+\cdots\right\} \\
& +\left\{f\left(R_{(n, i),(n-1, \mathcal{I}(1))} X, B\right) J_{\mathcal{I}(1)}+\left(1-f\left(R_{(n, i),(n-1, \mathcal{I}(1))} X, B\right)\right) \times J_{\mathcal{I}(2)}\right. \\
& +\cdots\} \\
& +T\left\{\left(1-f\left(R_{(n, i),(n-1, \mathcal{I}(1))} X, B\right) \times\left(1-f\left(R_{(n, i),(n-1, \mathcal{I}(2))} X, B\right)\right) \cdots\right\}\right.
\end{aligned}
$$

To see this, note that if the transmission is successful, then the delay from node $(n, i)$ to the next relay-set is $\frac{\text { packet size }}{B}$. The probability of experiencing this delay is given in the first and second lines. Furthermore, if transmission is successful, it experiences an expected additional delay of $J_{(n-1, j)}$. However, the node in the next relay-set that transmits depends on which node receives the packet and its relative values of $J$. Specifically, if node $(n-1, I(1))$ decodes the packet, a delay of $J_{(n-1, I(1))}$ will be experienced. If the packet does not reach node $(n-1, I(1))$, but does reach $(n-1, I(2))$, then a delay of $J_{(n-1, I(2))}$ is expected. The expected 
delay is given in the third and fourth lines. The expected delay due to retransmission is given in the last line. Once $J_{(n, i)}(B)$ is determined, we define $J_{(n, i)}=\min _{B} J_{(n, i)}(B)$. And, the node with the smallest $J_{(n, i)}$ transmits the packet.

In the simulations shown, $T=100$. However, different values of $T$ are possible and can result in some difference in performance. Specifically, if $T$ is very large, then, in order to make $J_{(n, i)}$ small, $f\left(R_{(n, i),(n-1, \mathcal{I}(1))}, B\right)$ will need to be very close to one. Hence, a conservative bit-rate will be selected. On the other hand, if $T$ is smaller, then the penalty of a transmission error is not so great and the bit-rate can be increased. Thus, $T$ acts much like a constraint on the transmission error probability. While the problem can also be framed so that the transmission error probability is fixed, in some settings, $T$ may be a more intuitive parameter than the transmission error probability.

The right-hand plot in Figure 2 shows the performance under this selection metric. It is assumed that the least-hop approach used a fixed bit-rate. In the idealized and implemented urban cases, the delay is reduced by a factor of between 3 and 14 . Note that the plot shows a small difference between the idealized urban and implemented urban cases. This contrasts the previous metrics where the implemented case gave better performance than the idealized case. The reason for this is that this selection metric does account for the possibly of opportunistic relaying.

\subsection{Minimizing the Total Transmission Power Sub- ject to per Link Channel Gain Constraint}

Here we define $J_{(n, i)}$ to be the total power required to deliver a packet from node $(n, i)$ to the destination while meeting per link channel gain constraint. Then

$$
J_{(n, i)}=\min _{(n-1, j)} \frac{C H^{*}}{R_{(n, i),(n-1, j)}}+J_{(n-1, j)}
$$

where $C H^{*}$ is the per link received power constraint. Note that the actual received signal power is $R_{(n, i),(n-1, j)} \times X$, where $X$ is the transmission power. Thus, if the transmitted power is $X=\frac{C H^{*}}{R_{(n, i),(n-1, j)}}$, then the received power constraint will be met.

In the idealized cases, the total transmission power is the value of $J$ at the source. In the implementation, each transmission power is summed. As in Section 4.1, (3) does not account for the possibly of opportunistic relaying and so the implementation shows better performance than the idealized case. 

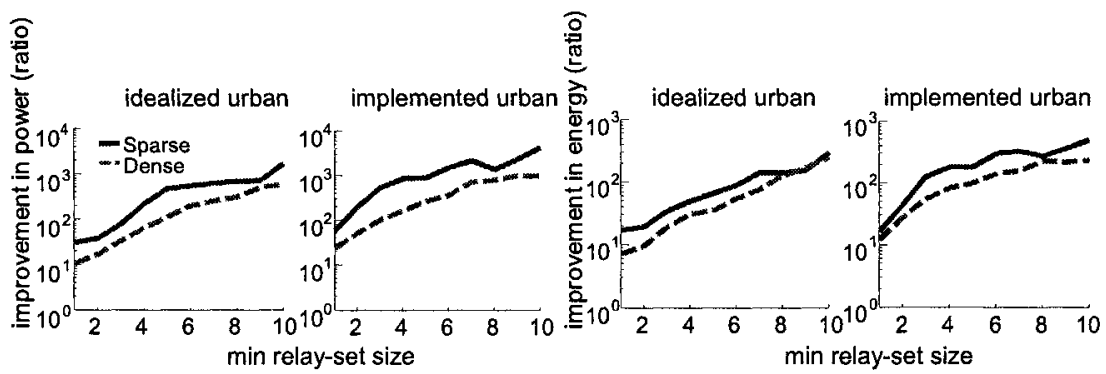

Figure 3. Left : Min power. The average ratio of the total end-to-end transmission power of least-hop routing to the total end-to-end transmission power with BSP. Right : Min energy. The average ratio of the total end-to-end transmission energy of least-hop routing to the total end-to-end transmission energy with BSP.

The left-hand plot in Figure 3 compares the performance of the BSP to least-hop in the different scenarios. Here we see that BSP yields dramatic performance improves over least-hop routing.

\subsection{Minimum Total Energy}

Let $J_{(n, i)}$ be the expected energy required to delivery the packet to the destination from node $(n, i)$. Furthermore, let $J_{(n, i)}(B, X)$ be the expected total energy required to deliver the packet from node $(n, i)$ to the destination if node $(n, i)$ transmits at bit-rate $B$ and with transmission power $X$. Then

$$
\begin{aligned}
& J_{(n, i)}(B, X)=X \frac{\text { packet size }}{B}\left\{f\left(R_{(n, i),(n-1, \mathcal{I}(1))} X, B\right)\right. \\
& \left.+\left(1-f\left(R_{(n, i),(n-1, \mathcal{I}(1))} X, B\right)\right) f\left(R_{(n, i),(n-1, \mathcal{I}(2))} X, B\right)+\cdots\right\} \\
& +\left\{f\left(R_{(n, i),(n-1, \mathcal{I}(1))} X, B\right) J_{\mathcal{I}(1)}+\left(1-f\left(R_{(n, i),(n-1, \mathcal{I}(1))} X, B\right)\right) \times J_{\mathcal{I}(2)}\right. \\
& +\cdots\} \\
& +M\left\{\left(1-f\left(R_{(n, i),(n-1, \mathcal{I}(1))} X, B\right)\right) \times\left(1-f\left(R_{(n, i),(n-1, \mathcal{I}(2))} X, B\right)\right) \cdots\right\}
\end{aligned}
$$

where $M$ is a parameter that represents the energy required to retransmit the packet due to transport layer retransmission. As in the minimum end-to-end delay metric examined in Section $4.2, M$ is set to a large value and can be used to control the probability of transmission error. The minimum energy selection metric can also be posed as a minimum energy with a constraint on the transmission error probability. Once $J_{(n, i)}(B, X)$ is known, then $J_{(n, i)}$ is found via $J_{(n, i)}=$ $\min _{B, X} J_{(n, i)}(B, X)$. 
In the idealized cases, the value of $J$ at the source is the total energy, but for the implementation, the total energy is found by summing the transmission power divided by the bit-rate. For the least-hop case, it is assumed that the bit-rate and transmission power is fixed. The total energy is found by computing $J_{(n, i)}$ but where the relay-sets are collapsed to the least-hop path. Note that this metric does account for the possibly of opportunistic relaying.

The right-hand plot in Figure 3 shows the performance under the minimum energy metric. The two cases are able to achieve dramatic reduction in energy, often well over an order of magnitude.

\section{Conclusion}

This paper examined several node selection metrics for the best-select protocol (BSP), considering urban idealized and implemented BSP. It is found that BSP can be used to increase performance in a number of ways. While the exact improvement depends on the environment and on the metric, improvements of a factor of 5 to as high as 1000 is not uncommon.

\section{Disclaimer}

The views and conclusions contained in this document are those of the authors and should not be interpreted as representing the official policies, either expressed or implied, of the Army Research Laboratory or the U. S. Government.

\section{References}

Bohacek, S., Hespanha, J., Lee, J., Lim, C., and Obraczka, K. (2002). Enhancing security via stochastic routing. In Proc. Of the 11th IEEE Int. Conf. On Comput. Communications and Networks.

Bohacek, S., Ilic, A., and Sridhara, V. (2005). On the predictability of link lifetimes in urban MANETs. In 3rd Intl. Symposium on Modeling and Optimization in Mobile, Ad Hoc, and Wireless Networks.

Luo, J., Blum, R. S., Greenstein, L. J., Cimini, L. J., and Haimovich, A. M. (2004). New approaches for cooperative use of multiple antennas in ad hoc wireless networks. In Proceedings of the IEEE Vehicular Technology Conference (VTC '04Fall).

Marina, M. K. and Das, S. R. (2003). Ad hoc on-demand multipath distance vector routing. Technical report, SUNY - Stony Brook.

Scalable Network Technologies (2005). The QualNet simulator http://www.qualnet.com/.

Sendonaris, A., Erkip, E., and Aazhang, B. (2003a). User cooperation diversity - Part I: System description. IEEE Transactions on Communications, 51:1927-1938.

Sridhara, V., Kim, J., and Bohacek, S. (2005). Models and methodologies for simulating mobile ad- hoc networks. In MobiWac.

Zhao, B. and Valenti, M. C. (2005). Practical relay networks: A generalization of hybrid-ARQ. IEEE Journal on Selected Areas in Communications, 23:7-18. 